\title{
Synthesis and potential cytotoxic activity of some new benzoxazoles, imidazoles, benzimidazoles and tetrazoles
}

SUBRAMANIYAN ARULMURUGAN HELEN P. KAVITHA*

Research Department of Chemistry SRM University, Ramapuram, Chennai Tamil Nadu, India

Accepted January 24, 2013
The present work deals with the synthesis of some novel heterocyclic compounds such as benzoxazoles 2, 7, 13 and 19, imidazoles 3, 8, 14 and 20, benzimidazoles 4, 9, 15 and 21, and tetrazoles 10, 16, and 22. The synthesized compounds were characterized by IR, ${ }^{1} \mathrm{H}$ NMR, mass spectrometry and elemental analysis. The compounds were evaluated for cytotoxicity against human cancer cell lines such as MCF-7 (breast cancer) and HT-29 (colon cancer) by the MTT assay method. Among the tested compounds, 4,4'-sulfonylbis( $N$-(2- $(1 H$-benzo $[d]$ imidazol-2-yl)ethyl)aniline (9), N-bis(2-(benzo[d]oxazol-2-yl)-ethyl)6-phenyl-1,3,5-triazine-2,4-diamine (13), N-bis(2-(1H-benzo[d]imidazol-2-yl)ethyl)-6-phenyl-1,3,5-triazine-2,4-diamine (15) and $N$-tris(2-1H-benzo[d]imidazol-2-yl)ethyl)1,3,5-triazine-2,4,6-triamine (21) showed potent cytotoxicity.

Keywords: benzimidazoles, imidazoles, benzoxazoles, tetrazoles, synthesis, cytotoxicity, MTT assay

Tumor can be defined as an abnormal mass of tissue, the growth of which exceeds and is uncoordinated with that of normal tissue and continues in the same manner after cessation of the stimuli that have initiated it (1). Control of disseminated tumor growth by systemically active chemotherapeutic agents remains a major challenge for cancer chemotherapy despite decades of focused efforts. Although there has been some notable success with certain forms of cancer, drug therapy has only a limited impact upon the three major killers: carcinoma of the lung, breast, and colorectal system (2).

Heterocyclic compounds are rich source of diverse physical, chemical and biological properties (3). Good therapeutic properties of the imidazole related drugs have encouraged medicinal chemists to synthesize a large number of novel chemotherapeutic agents. Medicinal properties of imidazoles include anticancer, inhibiting $\beta$-lactamase, 20-HETE (20-hydroxy-5,8,11,14-eicosatetraenoic acid) synthase, carboxypeptidase, heme oxygenase, antiaging, anticoagulating, anti-inflammatory, antibacterial, antifungal, antiviral,

\footnotetext{
* Correspondence; e-mail: helenkavithap@yahoo.co.in
} 
antitubercular, antidiabetic and antimalarial activities (4). Benzimidazoles are heterocyclic compounds having various biological activities (5), among which albendazole, mebendazole and thiabendazole are widely used anthelmintic drugs. Furthermore, benzimidazole compounds are also reported to possess biological activities such as inhibition of the angiopoietin receptor TIE-2 and tyrosine kinase receptor VEGFR-2 (vascular endothelial growth factor receptor-2), antitumor activity, thrombopoietin receptor agonistic activity, gamma-aminobutyric acid (GABA) agonistic activity, antimicrobial activity, topoisomerase inhibition, neuropeptide $\mathrm{Y} 1$ receptor antagonistic activity and inhibition of angiotensin II. Tetrazoles can act as pharmacophores for the carboxylate group and increase its utility $(6,7)$. Angiotensin II blockers often contain tetrazoles such as losartan and candesartan. A well-known tetrazole is dimethyl thiazolyl diphenyl tetrazolium salt (MTT), which is used in the MTT assay to quantify the respiratory activity of live cells in cell cultures, although it kills the cells in the process. Recent observations suggest that substituted benzoxazoles and related heterocycles possess potential activity with lower toxicity in the chemotherapeutic approach in man (8). Careful literature survey revealed that targets containing benzoxazole moiety, either isolated from plants or accessed by total synthesis, have remarkable biological activities, such as antimicrobial, antihistaminic, antiparasitic, herbicidal, antiallergic and antihelmintic activities. They were screened for cytotoxic activity by the MTT assay.

\section{EXPERIMENTAL}

Melting points were determined with a digital melting point apparatus (LAB India MR-VIS, India) and are uncorrected. Elemental analyses were performed on a Perkin-Elmer 2400 analyzer (Perkin-Elmer, USA) and were found within $\pm 0.4 \%$ of the theoretical values. FTIR spectra were recorded on a Shimadzu FT-IR model spectrophotometer (Japan). ${ }^{1} \mathrm{H}$ NMR spectra were recorded in DMSO on a Bruker AV III $500 \mathrm{MHz}$ (USA) using TMS as internal standard. Mass spectra were recorded on a JEOL GCmate instrument (Japan). The purity of the compounds was checked by TLC on pre-coated silica gel $\mathrm{G}\left(\mathrm{HF}_{254}\right)$ aluminium plates (Merck, USA) using chloroform/methanol (4:1) and visualized in a UV chamber.

Compounds 3-(4-(2-methyl-4-oxoquinazolin-3(4H)-yl)phenylamino)propanenitrile (1), 3,3'-(4,4'-sulfonylbis(4,1-phenylene)bis(azanediyl))dipropanenitrile (6), 3,3'-(6-phenyl-1,3,5-triazine-2,4-diyl)bis(azanediyl)dipropanenitrile (12) and 3,3,3',1,3,5-triazine-2,4,6-triyl)tris(azanediyl)tripropanenitrile (18) were prepared from primary amine by treatment with acrylonitrile and Triton B following reported literature (9).

Syntheses of benzoxazoles, imidazoles and benzimidazoles. - 3-(4-(2-(benzo[d]oxazol-2-yl)-ethylamino)phenyl)-2-methylquinazolin-4(3H)-one (2), 4,4'-sulfonylbis( $N$-(2-(benzo[ $d]$ oxazol-2-yl)ethyl)aniline (7), $N$-bis(2-(benzo[d]oxazol-2-yl)ethyl)-6-phenyl-1,3,5-triazine-2,4-diamine (13), $N$-tris(2-(benzo[d]oxazol-2-yl)ethyl)-1,3,5-triazine-2,4,6-triamine (19), 3-(4-(2-((1H-imidazol-2-yl)ethylamino)phenyl-2-methylquinazolin-4(3H)-one (3), 4,4'sulfonylbis( $\mathrm{N}$-(1H-imidazol-2-yl)ethylaniline (8), $\mathrm{N}$-bis(2-1H-imidazol-2-yl)ethyl)-6-phenyl1,3,5-triazine-2,4-diamine (14), $\quad N$-tris(2-1H-imidazol-2-yl)ethyl)-1,3,5-triazine-2,4,6-triamine (20), 3-(4-(2-(1H-benzo[ $d]$ imidazol-2-yl)ethylamino)phenyl)-2-methylquinazolin- 
S. Arulmurugan and H. P. Kavitha: Synthesis and potential cytotoxic activity of some new benzoxazoles, imidazoles, benzimidazoles and tetrazoles, Acta Pharm. 63 (2013) 253-264.

Table I. Physical and analytical data of synthesized compounds

\begin{tabular}{|c|c|c|c|c|c|c|c|}
\hline \multirow{2}{*}{ Compd. } & \multirow{2}{*}{ Mol. formula } & \multirow{2}{*}{$M_{\mathrm{r}}$} & \multirow{2}{*}{$\begin{array}{c}\text { Yield } \\
(\%)\end{array}$} & \multirow{2}{*}{ M. p. $\left({ }^{\circ} \mathrm{C}\right)$} & \multicolumn{3}{|c|}{ Elemental analysis (calcd./found, \%) } \\
\hline & & & & & C & $\mathrm{H}$ & $\mathrm{N}$ \\
\hline \multirow{2}{*}{2} & \multirow{2}{*}{$\mathrm{C}_{24} \mathrm{H}_{20} \mathrm{~N}_{4} \mathrm{O}_{2}$} & \multirow{2}{*}{396.16} & \multirow{2}{*}{74} & \multirow{2}{*}{$310-313$} & 72.71 & 5.08 & 14.13 \\
\hline & & & & & 72.77 & 5.18 & 14.17 \\
\hline \multirow{2}{*}{3} & \multirow{2}{*}{$\mathrm{C}_{20} \mathrm{H}_{19} \mathrm{~N}_{5} \mathrm{O}$} & \multirow{2}{*}{345.16} & \multirow{2}{*}{77} & \multirow{2}{*}{$257-259$} & 69.55 & 5.54 & 20.28 \\
\hline & & & & & 69.61 & 5.36 & 20.32 \\
\hline \multirow{2}{*}{4} & \multirow{2}{*}{$\mathrm{C}_{24} \mathrm{H}_{21} \mathrm{~N}_{5} \mathrm{O}$} & \multirow{2}{*}{395.17} & \multirow{2}{*}{75} & \multirow{2}{*}{ 299-301 } & 72.89 & 5.35 & 17.71 \\
\hline & & & & & 72.91 & 5.41 & 17.66 \\
\hline \multirow{2}{*}{6} & \multirow{2}{*}{$\mathrm{C}_{18} \mathrm{H}_{18} \mathrm{~N}_{4} \mathrm{O}_{2} \mathrm{~S}$} & \multirow{2}{*}{354.12} & \multirow{2}{*}{68} & \multirow{2}{*}{ 99-102 } & 61.00 & 5.12 & 15.81 \\
\hline & & & & & 61.13 & 5.17 & 15.90 \\
\hline 7 & $\mathrm{C}_{2} \mathrm{H}_{2} \mathrm{~N}_{2} \mathrm{O}_{5} \mathrm{~s}$ & 53817 & 72 & $240-242$ & 66.90 & 4.87 & 10.40 \\
\hline 7 & $C_{30} \mathrm{I}_{26^{1}} \mathrm{~N}_{4}-\mathrm{U}_{4}$ & 538.17 & 12 & $240-242$ & 66.63 & 4.89 & 10.22 \\
\hline 8 & $\mathrm{C}_{2} \mathrm{H}_{2} \mathrm{NO}_{2}$ & 43617 & 71 & 197-200 & 60.53 & 5.54 & 19.25 \\
\hline 0 & $22^{11} 4^{1}{ }_{6} U^{3}$ & 430.17 & 11 & $197-200$ & 60.61 & 5.63 & 19.27 \\
\hline 9 & $\mathrm{C}_{3} \mathrm{H}_{20} \mathrm{~N}_{6} \mathrm{O}_{2} \mathrm{~S}$ & 53620 & 76 & $230-232$ & 67.14 & 5.26 & 15.66 \\
\hline y & 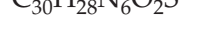 & 536.20 & 16 & $230-232$ & 66.95 & 5.18 & 15.61 \\
\hline 10 & $\mathrm{C}_{18} \mathrm{H}_{20} \mathrm{~N}_{10} \mathrm{O}_{2} \mathrm{~S}$ & 440.15 & 69 & $99-103$ & 49.08 & 4.58 & 31.80 \\
\hline & $18^{1}+20^{1} \cdot 10-v_{2}$ & 440.15 & 09 & (2) & 49.17 & 4.61 & 31.97 \\
\hline 12 & $\mathrm{C}_{15} \mathrm{H}_{15} \mathrm{~N}_{7}$ & 293.33 & 64 & 99-102 & 61.42 & 4.15 & 33.43 \\
\hline & & & & & 61.56 & 4.21 & 33.48 \\
\hline 13 & $\mathrm{C}_{27} \mathrm{H}_{2} \mathrm{~N}_{7} \mathrm{O}_{2}$ & 47719 & 67 & $167-169$ & 67.91 & 4.85 & 20.53 \\
\hline 10 & $27^{1}{ }^{2} 3^{1} v_{7} U_{2}$ & $4 / 7.19$ & br & $16 /-169$ & 67.96 & 4.83 & 20.47 \\
\hline 14 & $\mathrm{C}_{19} \mathrm{H}_{21} \mathrm{~N}_{9}$ & 375.19 & 69 & 197-199 & 60.78 & 5.64 & 33.58 \\
\hline & $19 \times 21^{1}=4$ & & ( & 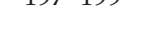 & 60.74 & 5.67 & 33.61 \\
\hline 15 & $\mathrm{C}_{27} \mathrm{H}_{25} \mathrm{~N}_{9}$ & 475.22 & 71 & $219-220$ & 68.19 & 5.30 & 26.51 \\
\hline & $-27^{1}-25^{1}$ v & $4 / 5.22$ & 11 & $219-2 \angle 0$ & 68.12 & 5.34 & 26.44 \\
\hline 16 & $\mathrm{C}_{15} \mathrm{H}_{17} \mathrm{~N}_{13}$ & 37917 & 68 & 180-200 & 47.49 & 4.52 & 48.00 \\
\hline & $-15+17+413$ & (3/9.17 & 00 & $100-200$ & 47.44 & 4.49 & 48.04 \\
\hline 18 & $\mathrm{C}_{12} \mathrm{H}_{15} \mathrm{~N}_{9}$ & 285.15 & 63 & 95-97 & 50.52 & 5.30 & 44.18 \\
\hline & & & & & 50.49 & 5.27 & 44.25 \\
\hline 19 & $\mathrm{C}_{20} \mathrm{H}_{27} \mathrm{~N}_{0} \mathrm{O}_{3}$ & 561.22 & 72 & 138-139 & 64.16 & 4.85 & 22.45 \\
\hline & 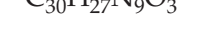 & & & $130-139$ & 64.12 & 4.82 & 22.41 \\
\hline 20 & $\mathrm{C}_{18} \mathrm{H}_{24} \mathrm{~N}_{12}$ & 408.22 & 66 & $210-212$ & 52.93 & 5.92 & 41.65 \\
\hline & & & & & 52.89 & 5.97 & 41.69 \\
\hline 21 & $\mathrm{C}_{20} \mathrm{H}_{20} \mathrm{~N}_{12}$ & 558 & 75 & $184-185$ & 64.50 & 5.41 & 30.09 \\
\hline 21 & $30^{1+} 30^{1-12}$ & 558 & 10 & $184-185$ & 64.46 & 5.32 & 30.18 \\
\hline 22 & $\mathrm{C}_{12} \mathrm{H}_{18} \mathrm{~N}_{18}$ & 414.20 & 73 & $248-250$ & 34.78 & 4.38 & 60.84 \\
\hline & & & & & 34.73 & 4.42 & 60.87 \\
\hline
\end{tabular}


S. Arulmurugan and H. P. Kavitha: Synthesis and potential cytotoxic activity of some new benzoxazoles, imidazoles, benzimidazoles and tetrazoles, Acta Pharm. 63 (2013) 253-264.

Table II. Spectral data of synthesized compounds

\begin{tabular}{lcl}
\hline Compd. & IR $(\mathrm{KBr})\left(v, \mathrm{~cm}^{-1}\right)$ & \multicolumn{1}{c}{${ }^{1} \mathrm{H}$ NMR $\left(\mathrm{DMSO}_{-} \mathrm{d}_{6}\right)(\delta, \mathrm{ppm})$} \\
\hline 2 & $3306(\mathrm{C}-\mathrm{H}), 2921\left(\mathrm{CH}_{2}\right)$, & $2.08\left(\mathrm{~s}, 3 \mathrm{H}, \mathrm{CH}_{3}\right), 7.53(\mathrm{~s}, 12 \mathrm{H}, \mathrm{Ar}-\mathrm{H})$, \\
& $1507(\mathrm{C}=\mathrm{N}), 3400(-\mathrm{NH})$ & $3.34\left(\mathrm{~s}, 2 \mathrm{H}, \mathrm{CH}_{2}\right), 3.02\left(\mathrm{~s}, 2 \mathrm{H}, \mathrm{CH}_{2}\right)$ \\
& & \\
3 & $3221(\mathrm{C}-\mathrm{H}), 2926\left(\mathrm{CH}_{2}\right)$, & $6.7-7.5(\mathrm{~m}, 8 \mathrm{H}, \mathrm{Ar}-\mathrm{H}), 3.37(\mathrm{~s}, 2 \mathrm{H}$, \\
& $1609(\mathrm{C}=\mathrm{N}), 3346(-\mathrm{NH})$ & $\left.\mathrm{CH}_{2}\right), 2.48\left(\mathrm{~s}, 3 \mathrm{H}, \mathrm{CH}_{3}\right), 8.2(\mathrm{~s}, \mathrm{NH})$
\end{tabular}

4

$3055(\mathrm{C}-\mathrm{H}), 2926\left(\mathrm{CH}_{2}\right), \quad 6-6.4(\mathrm{~m}, 8 \mathrm{H}, \mathrm{Ar}-\mathrm{H}), 3.6$ (s, 2H, $\left.1627(\mathrm{C}=\mathrm{N}), 3315(-\mathrm{NH}) \quad \mathrm{CH}_{2}-\mathrm{C}\right), 2.9\left(\mathrm{~s}, 2 \mathrm{H}, \mathrm{CH}_{2}\right), 2.48(\mathrm{~s}, 3 \mathrm{H}$, $\left.-\mathrm{N}=\mathrm{C}-\mathrm{CH}_{3}\right), 8.1$ (s, $\mathrm{NH}$ benzimidazole) $3100(\mathrm{C}-\mathrm{H}), 2929\left(\mathrm{CH}_{2}\right), \quad 7.31-7.5(\mathrm{~m}, 8 \mathrm{H}$, benzoxazole $), 7.5$ $1591(\mathrm{C}=\mathrm{N}), 3350(-\mathrm{NH}) \quad(\mathrm{d}, 4 \mathrm{H}, \mathrm{J}=11.5, \mathrm{Ar}-\mathrm{H}), 6.57-6.58(\mathrm{~d}$, $4 \mathrm{H}, \mathrm{J}=8.5, \mathrm{Ar}-\mathrm{H}), 3.58\left(\mathrm{~s}, 2 \mathrm{H}, \mathrm{CH}_{2}\right)$, $2.89\left(\mathrm{~s}, 2 \mathrm{H},-\mathrm{CH}_{2}\right) 7.95(\mathrm{~s}, 1 \mathrm{H}, \mathrm{NH})$ $3085(\mathrm{C}-\mathrm{H}), 2928\left(\mathrm{CH}_{2}\right), \quad 6.56-6.58(\mathrm{~d}, 4 \mathrm{H}, \mathrm{J}=8.5, \mathrm{Ar}-\mathrm{H})$, $1634(\mathrm{C}=\mathrm{N}), 3421(-\mathrm{NH}) \quad 7.42-7.44(\mathrm{~d}, 4 \mathrm{H}, \mathrm{J}=8.5, \mathrm{Ar}-\mathrm{H}), 3.38$ (s, $\left.2 \mathrm{H},-\mathrm{CH}_{2}-\mathrm{N}\right), 3.8(\mathrm{~s}, \mathrm{C}-\mathrm{NH}), 5.98$ (s, $4 \mathrm{H}, \mathrm{CH}_{2}$ imidazole ring), 8.2 (s, NH, imidazole)

$3223(\mathrm{C}-\mathrm{H}), 2929\left(\mathrm{CH}_{2}\right)$, 6.58-6.69 (m, 4H, Ar-H), 7.1-7.5 $1592(\mathrm{C}=\mathrm{N}), 3360(-\mathrm{NH}) \quad(\mathrm{m}, 4 \mathrm{H}, \mathrm{Ar}-\mathrm{H}), 3.30\left(\mathrm{~s}, 2 \mathrm{H}, \mathrm{CH}_{2}\right)$, $2.80\left(\mathrm{~s}, 2 \mathrm{H}, \mathrm{CH}_{2}\right), 8.0(\mathrm{~s}, 1 \mathrm{H}, \mathrm{NH}$ benzimidazole)

$3098(\mathrm{C}-\mathrm{H}), 3005\left(\mathrm{CH}_{2}\right), \quad 7.5-7.8(\mathrm{~m}, 4 \mathrm{H}, \mathrm{Ar}-\mathrm{H}), 6.68-.69$ (d, $1602(\mathrm{C}=\mathrm{N}), 3426(-\mathrm{NH}), 4 \mathrm{H}, J=18, \mathrm{Ar}-\mathrm{H}), 3.38\left(\mathrm{~s}, 2 \mathrm{H},-\mathrm{CH}_{2}\right)$, $1424(-\mathrm{N}=\mathrm{N}), 1225$ $2.8\left(\mathrm{~s}, 2 \mathrm{H}, \mathrm{CH}_{2}\right) 8.3(\mathrm{~s}, 1 \mathrm{H}, \mathrm{NH})$ $(-\mathrm{N}-\mathrm{N}=\mathrm{N}), 1102$ and 11455.7 (s, NH tetrazole) (tetrazole ring) 3050 (C-H), $2921\left(\mathrm{CH}_{2}\right), \quad 7.5$ (s, 12H, Ar-H), 3.4 (s, 2H, $\left.\mathrm{CH}_{2}\right)$, $1529(\mathrm{C}=\mathrm{N}), 3400(-\mathrm{NH}) \quad 2.8\left(\mathrm{~s}, 2 \mathrm{H},-\mathrm{CH}_{2}\right)$

$3005(\mathrm{C}-\mathrm{H}), 2643\left(\mathrm{CH}_{2}\right), \quad 3.4\left(\mathrm{~s}, 2 \mathrm{H},-\mathrm{CH}_{2}\right), 2.8\left(\mathrm{~s}, 2 \mathrm{H}, \mathrm{CH}_{2}\right)$, $1697(\mathrm{C}=\mathrm{N}), 3417(-\mathrm{NH}) . \quad 5.72\left(\mathrm{~s}, 4 \mathrm{H},-\mathrm{CH}_{2}\right.$ imidazole ring), 8.1 (s, NH, imidazole), 7.5 (s, 8H, Ar-H) $1590(\mathrm{C}=\mathrm{N}), 3397(-\mathrm{NH}), \quad 4(\mathrm{~s}, 1 \mathrm{H}, \mathrm{NH}), 7.3-8.5(\mathrm{~s}, 5 \mathrm{H}, \mathrm{Ar}-\mathrm{H})$, $1430(-\mathrm{N}=\mathrm{N}), 1231 \quad 5.5(\mathrm{~s}, 1 \mathrm{H}, \mathrm{NH}$ tetrazole ring $)$

$(-\mathrm{N}-\mathrm{N}=\mathrm{N}), 1099$ and 1169 $3.42\left(\mathrm{~s}, 2 \mathrm{H}, \mathrm{CH}_{2}\right), 2.89\left(\mathrm{~s}, 2 \mathrm{H}, \mathrm{CH}_{2}\right)$; $4.5(\mathrm{~s}, 1 \mathrm{H},-\mathrm{NH}), 7.45$ (s, 12H, Ar-H), $12.33(\mathrm{~s}, 1 \mathrm{H},-\mathrm{NH}$ benzimidazole) (tetrazole ring)

394.54 (M-1, 70), 294.91

(72), $279.98(75), 269.23$

(78), $206.26(100)$

537.92 (M-1, 17), 425.06

(6), 126.84 (100), 254.62

(37), 182.86 (52)

435.72 (M-1, 6), 423.75

(20), 247.61 (50), 101.76

(100), 139.77 (37)

535.77 (M-1, 7), 252.77

(15), 148.89 (100), 122.88

$440.63\left(\mathrm{M}^{+}, 27\right), 260.60$ (100), 247.61 (77), 300.52

$478.76(\mathrm{M}+1,5), 442.31$ (25), 262.25 (74), 182.62 (100), 167.77 (63)

374.56 (M-1, 9), 172.23 (100), 164.67 (42)

$475.05\left(\mathrm{M}^{+}, 5\right), 442.32$

(16), 262.26 (37), 182.72 (67), 54.50 (100)

378.78 (M-1, 7), 262.26

(19), 247.30 (41), 182.72

(36), 167.79 (100), 121.98 (19)

$561.10\left(\mathrm{M}^{+}, 10\right), 550.32(8)$, 518.11 (15), 246.33 (100), 229.36 (25), 213.38 (17), 
S. Arulmurugan and H. P. Kavitha: Synthesis and potential cytotoxic activity of some new benzoxazoles, imidazoles, benzimidazoles and tetrazoles, Acta Pharm. 63 (2013) 253-264.

\begin{tabular}{|c|c|c|c|}
\hline 20 & $\begin{array}{l}3099(\mathrm{C}-\mathrm{H}), 2883\left(\mathrm{CH}_{2}\right) \\
1631(\mathrm{C}=\mathrm{N}), 3261(-\mathrm{NH})\end{array}$ & $\begin{array}{l}2.5\left(\mathrm{~s}, 2 \mathrm{H}, \mathrm{CH}_{2}\right), 3.4(\mathrm{~s}, 2 \mathrm{H}, \mathrm{CH} 2), 6 \\
(\mathrm{~s}, 1 \mathrm{H}, \mathrm{NH}), 7.9(\mathrm{~s}, 1 \mathrm{H}, \mathrm{NH} \text { imidazole })\end{array}$ & $\begin{array}{l}408.10\left(\mathrm{M}^{+}, 7\right), 393.34 \\
(5), 58.71(100), 201.11 \\
(72), 217.95(52)\end{array}$ \\
\hline 21 & $\begin{array}{l}3164(\mathrm{CH}), 2930\left(\mathrm{CH}_{2}\right) \\
1634(\mathrm{C}=\mathrm{N}), 3411(-\mathrm{NH})\end{array}$ & $\begin{array}{l}2.5\left(\mathrm{~s}, 2 \mathrm{H}, \mathrm{CH}_{2}\right), 3.4\left(\mathrm{~s}, 2 \mathrm{H}, \mathrm{CH}_{2}\right) \\
7.5(\mathrm{~s}, 12 \mathrm{H}, \mathrm{Ar}-\mathrm{H}), 12.53(\mathrm{~s}, 1 \mathrm{H},-\mathrm{NH} \\
\text { benzimidazole })\end{array}$ & $\begin{array}{l}558.10\left(\mathrm{M}^{+}, 5\right), 63.13 \\
(100), 175.29(17) \\
145.73(45)\end{array}$ \\
\hline & $\begin{array}{l}3205(\mathrm{C}-\mathrm{H}), 2939\left(\mathrm{CH}_{2}\right), \\
1655(\mathrm{C}=\mathrm{N}), 3408(-\mathrm{NH}), \\
1404(-\mathrm{N}=\mathrm{N}), 1225 \\
(-\mathrm{N}-\mathrm{N}=\mathrm{N}), 1101 \text { and } 1174 \\
\text { (tetrazole ring) }\end{array}$ & $\begin{array}{l}2.5\left(\mathrm{~s}, 2 \mathrm{H}, \mathrm{CH}_{2}\right), 3.4\left(\mathrm{~s}, 2 \mathrm{H}, \mathrm{CH}_{2}\right) \\
7.5-8.0(\mathrm{~s}, 5 \mathrm{H}, \mathrm{Ar}-\mathrm{H}), 5.5(\mathrm{~s}, 1 \mathrm{H}, \mathrm{NH} \\
\text { tetrazole ring) }\end{array}$ & $\begin{array}{l}414.13\left(\mathrm{M}^{+}, 11\right), 70.83 \\
(100), 251.43(32), 373.20 \\
(17), 110.75(38), 96.81 \\
(58)\end{array}$ \\
\hline
\end{tabular}

-4(3H)-one (4), 4,4'-sulfonylbis( $N$-(2-(1H-benzo[ $d]$ imidazol-2-yl)ethyl)aniline (9), $N$-bis(2-(1H-benzo[d]imidazol-2-yl)ethyl)-6-phenyl-1,3,5-triazine-2,4-diamine (15), N-tris $(2-1 H$ -benzo[d]imidazol-2-yl)ethyl)-1,3,5-triazine-2,4,6-triamine (21).

To a mixture of (3-(4-(2-methyl-4-oxoquinazolin-3(4H)-yl)phenylamino)propanenitrile (1) $(0.01 \mathrm{~mol}, 2.13 \mathrm{~g}), 3,3^{\prime}$-(4,4'-sulfonylbis(4,1-phenylene)bis(azanediyl))dipropanenitrile (6) (0.01 mol, $3.54 \mathrm{~g})$ or 3,3'-(6-phenyl-1,3,5-triazine-2,4-diyl)bis(azanediyl)dipropanenitrile (12) $(0.01 \mathrm{~mol}, 2.93 \mathrm{~g})$ and 3,3,3',1,3,5-triazine-2,4,6-triyl)tris(azanediyl)tripropanenitrile (18) $(0.01 \mathrm{~mol}, 2.85 \mathrm{~g})$ and $\mathrm{o}$-aminophenol $(0.01 \mathrm{~mol}, 1.09 \mathrm{~g})$ or ethylenediamine $(0.01 \mathrm{~mol}, 0.60 \mathrm{~g})$ or $o$-phenylenediamine $(0.01 \mathrm{~mol}, 1.08 \mathrm{~g}), 5 \mathrm{~mL}$ of conc. $\mathrm{HCl}$ was added and refluxed at $160^{\circ} \mathrm{C}$ on an oil bath for ten hours. The hydrochloride was precipitated after cooling for four hours. The precipitate was filtered and washed with an ethanol/ether mixture (1:5), suspended in acetone and made alkaline with a strong ammonia solution. Excess of water was added to liberate the base as a solid. The solid was filtered, washed with water and recrystallized from methanol to get benzoxazole or imidazole or benzimidazole by reacting the nitriles with ethylenediamine and $o$-phenylenediamine, respectively.

Syntheses of tetrazole $\left(4,4^{\prime}\right.$-sulfonylbis( $\mathrm{N}-(2-(1 \mathrm{H}-$ tetrazole-5-yl)ethyl)aniline) (10), N-bis(2-1H-tetrazol-5-yl)ethyl)-6-phenyl-1,3,5-triazine-2,4-diamine (16) and N-tris(2-(1H-tetrazol-5-yl)ethyl)-1,3,5-triazine-2,4,6-triamine (22).

To a mixture of compound (3-(4-(2-methyl-4-oxoquinazolin-3(4H)-yl)phenylamino)propanenitrile (1) $(0.01 \mathrm{~mol}, 2.13 \mathrm{~g}), 3,3^{\prime}$-(4,4'-sulfonylbis(4,1-phenylene)bis(azanediyl))dipropanenitrile (6) (0.01 mol, $3.54 \mathrm{~g}), 3,3$ '-(6-phenyl-1,3,5-triazine-2,4-diyl)bis(azanediyl)dipropanenitrile (12) (0.01 mol, $2.93 \mathrm{~g})$ or 3,3,3',1,3,5-triazine-2,4,6-triyl)tris(azanediyl)tripropanenitrile (18) $(0.01 \mathrm{~mol}, 2.85 \mathrm{~g})$, sodium azide $(0.01 \mathrm{~mol}, 0.65 \mathrm{~g})$, dimethylformamide $(10 \mathrm{~mL})$ and $\mathrm{NH}_{4} \mathrm{Cl}(0.1 \mathrm{~mol}, 5.3 \mathrm{~g})$ was placed in a $100-\mathrm{mL}$ round-bottomed flask. The content was heated in oil bath for seven hours at $125^{\circ} \mathrm{C}$. The solvent was removed at reduced pressure. The reaction mixture was dissolved in $100 \mathrm{~mL}$ distilled water and carefully acidified with dil. $\mathrm{HCl}(1: 1)(2 \mathrm{~mL})$ to make solution of $\mathrm{pH} 2$. The solution was cooled to $5^{\circ} \mathrm{C}$ in an ice bath. The product was isolated by filtration, washed with several portions of water and dried. The crude product was recrystallized from DMF.

Physicochemical and spectral data for the synthesized compounds are given in Tables I and II. Synthetic routes are presented in Schemes 1-4. 
S. Arulmurugan and H. P. Kavitha: Synthesis and potential cytotoxic activity of some new benzoxazoles, imidazoles, benzimidazoles and tetrazoles, Acta Pharm. 63 (2013) 253-264.

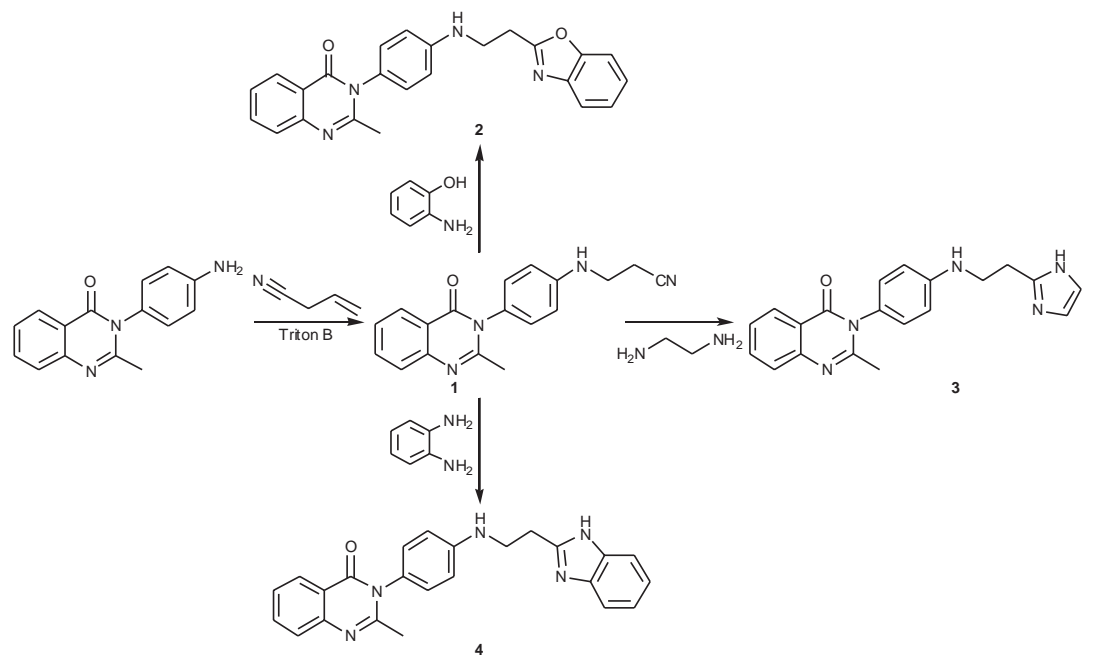

Scheme 1.

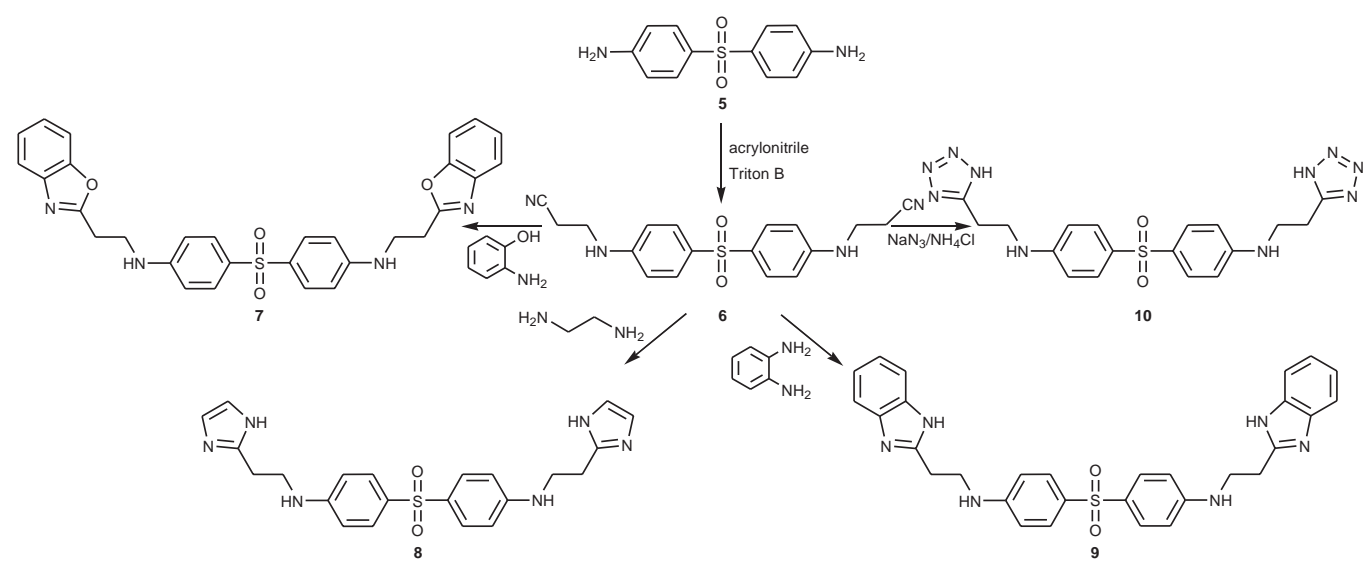

Scheme 2. 


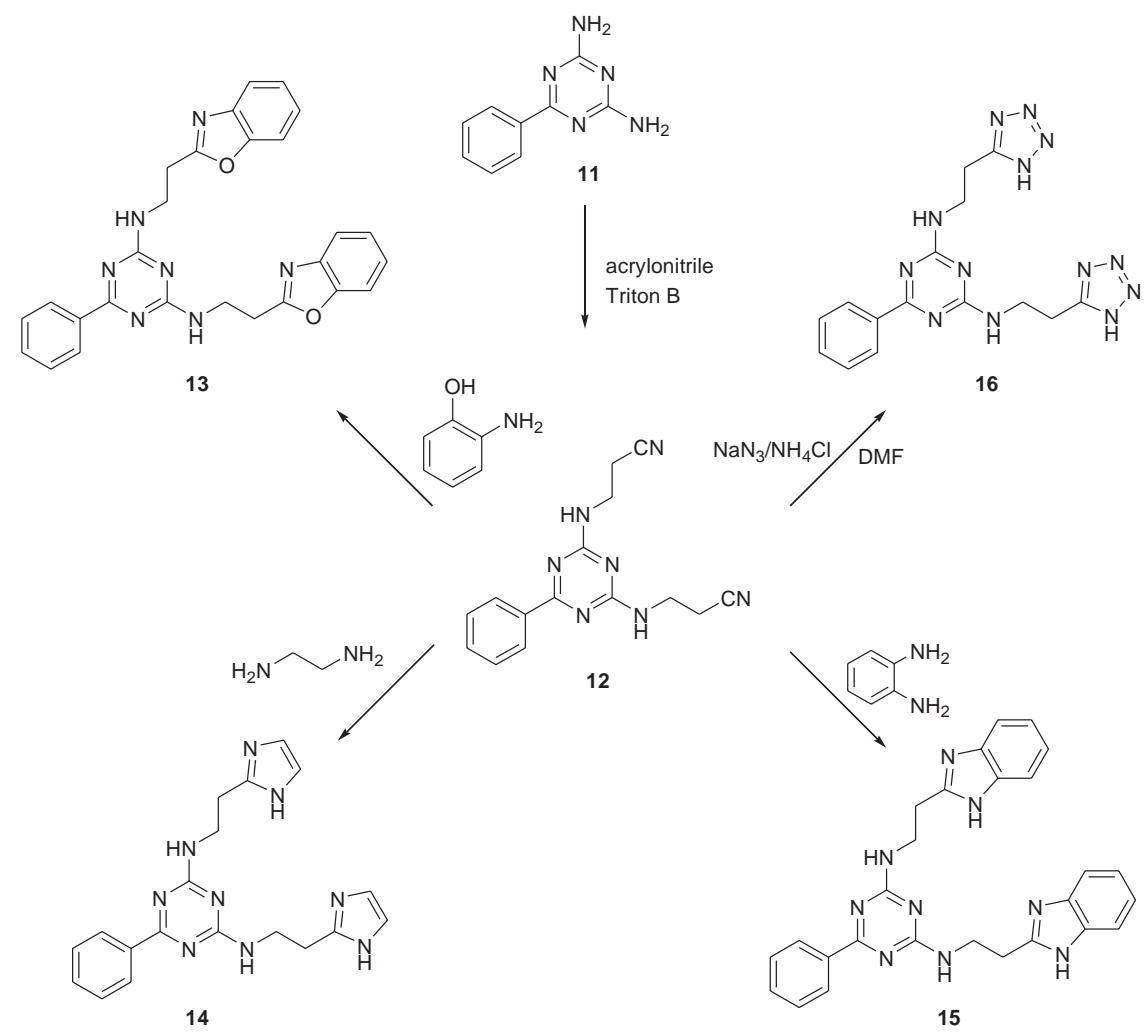

Scheme 3 .

\section{MTT assay}

The tumor cell growth inhibition activities of the newly synthesized compounds were assessed in vitro on the human cancer cell line MCF-7 (breast cancer) and HT-29 (colon cancer) obtained from the National Centre for Cell Sciences (NCCS), Pune, India. 5-Fluorouracil (Merck, Germany) was used as a standard.

Cultured cells were trypsinized and resuspended in growth medium. The cells were seeded at $1 \times 10^{5}$ cells per well in a 96-well plate and incubated for $24 \mathrm{~h}$ in a $\mathrm{CO}_{2}$ incubator. The cells were treated with different concentrations $(1,5,10,12.5,25,50,75$, 100,125 and $250 \mu \mathrm{g} \mathrm{mL}^{-1}$ ) of test compounds and then incubated for $24 \mathrm{~h}$ in the $\mathrm{CO}_{2}$ incubator. To each well, $10 \mu \mathrm{L}$ of MTT reagent was added and they were again incubated for $4 \mathrm{~h}$ at $37^{\circ} \mathrm{C}$. Each experiment was done in triplicate. The content of each well was transferred to a different Eppendorf tube and centrifuged at $2000 \mathrm{rpm}$ for 10 minutes. The pellets obtained were dissolved by adding $200 \mu \mathrm{L}$ of DMSO and were made up to $2 \mathrm{~mL}$. Absorbance was measured at $550 \mathrm{~nm}$ and the \% viability was calculated from the mean absorbance of the test compound in respect to the mean absorbance of the control. 


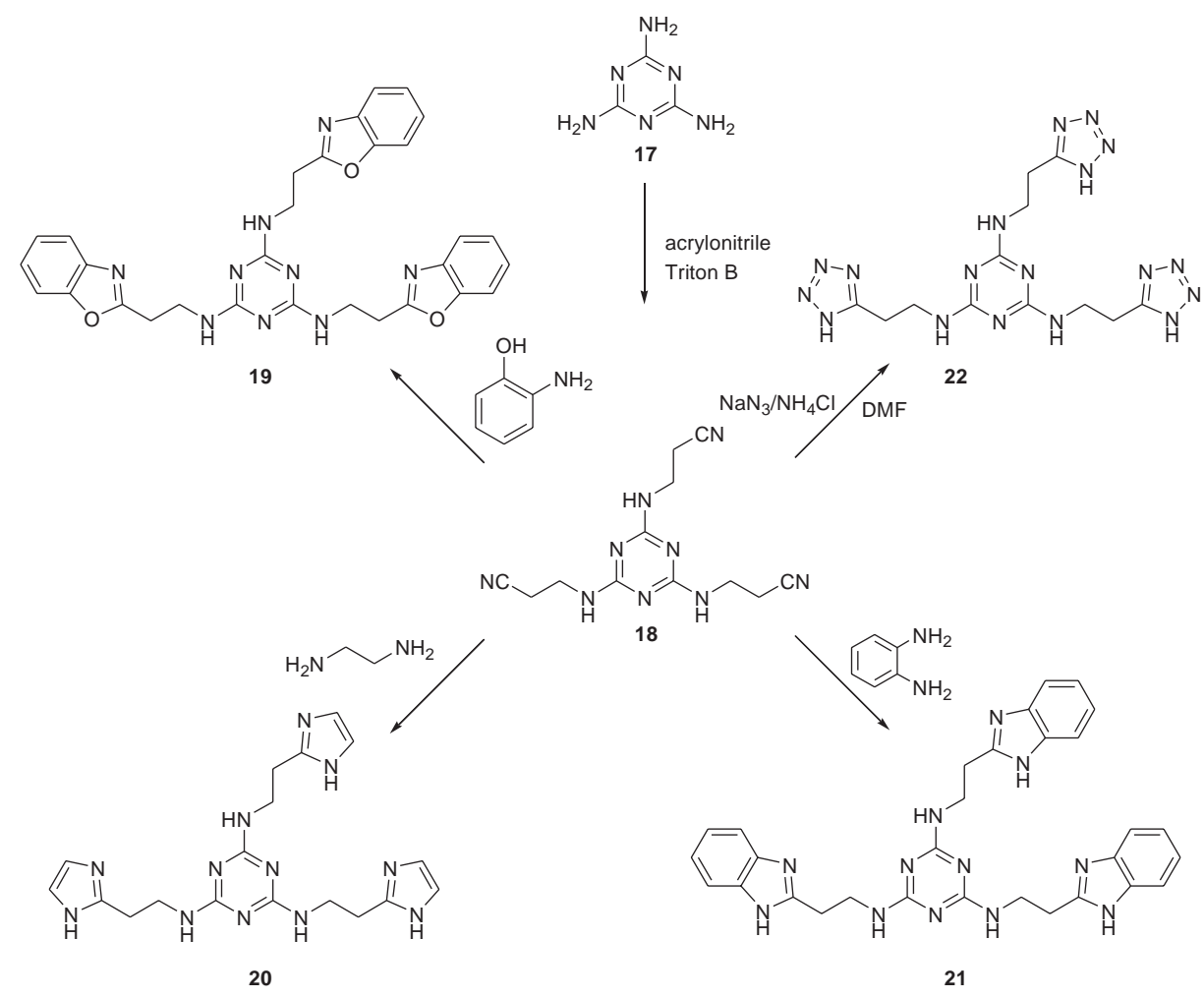

Scheme 4

The $I C_{50}$ values were reported as mean \pm standard deviation of three independent experiments. One-way analysis of variance (ANOVA) and Student $t$-test were used to compare data using the Graph Pad Prism 5.0 software at a $95 \%$ confidence limit.

\section{RESULTS AND DISCUSSION}

\section{Chemistry}

3-(4-(2-Methyl-4-oxoquinazolin-3(4H)-yl)phenylamino)propanenitrile (1) on treatment with $o$-aminophenol, ethylenediamine and $o$-phenylene diamine yielded the compounds 3-(4-(2-(benzo[ $d]$ oxazol-2-yl)ethylamino)phenyl)-2-methylquinazolin-4(3H)-one (2), 3-(4-(2-((1H-imidazol-2-yl)ethylamino)phenyl-2-methylquinazolin-4(3H)-one (3) and 3-(4-(2-(1H-benzo[d]imidazol-2-yl)ethylamino)phenyl)-2-methylquinazolin-4(3H)-one (4), respectively (Scheme 1). A similar reaction was carried out to synthesize compounds 7-9 (Scheme 2), 14-16 (Scheme 3) and 20-22 (Scheme 4) from compound 3,3'-(4,4'-sulfonylbis(4,1-phenylene)bis(azanediyl))dipropanenitrile (6), 3,3'-(6-phenyl-1,3,5-triazine-2,4- 
S. Arulmurugan and H. P. Kavitha: Synthesis and potential cytotoxic activity of some new benzoxazoles, imidazoles, benzimidazoles and tetrazoles, Acta Pharm. 63 (2013) 253-264.

-diyl)bis(azanediyl)dipropanenitrile (12) and 3,3,3',1,3,5-triazine-2,4,6-triyl)tris(azanediyl)tripropanenitrile (18) respectively. The structures of the compounds were confirmed by IR, ${ }^{1} \mathrm{H}$ NMR, mass spectra and elemental analysis.

Benzoxazoles (3-(4-(2-(benzo[d]oxazol-2-yl)ethylamino)phenyl)-2-methylquinazolin-4(3H)-one (2), 4,4'-sulfonylbis( $N$-(2-(benzo[d]oxazol-2-yl)ethyl)aniline) (7), $N$-bis(2-(ben zo[d]oxazol-2-yl)ethyl)-6-phenyl-1,3,5-triazine-2,4-diamine (13) and $N$-tris(2-(benzo[d]oxazol-2-yl)ethyl)-1,3,5-triazine-2,4,6-triamine (19) were prepared by treating compounds $\mathbf{1}$, 6, 12 and 18 with $o$-aminophenol in the presence of conc. $\mathrm{HCl}$ at $160{ }^{\circ} \mathrm{C}$. In general, infrared spectral data of all benzoxazoles $(2,7,13,19)$ revealed bands at 3350-3402 $(-\mathrm{NH})$, 2921-2929 $\left(\mathrm{CH}_{2}\right), 1507-1639(\mathrm{C}=\mathrm{N})$ and $3050-3306 \mathrm{~cm}^{-1}(\mathrm{CH})$. The ${ }^{1} \mathrm{H}$ NMR spectrum of the compounds showed a singlet at $\delta 3.4-3.58 \mathrm{ppm}$ which can be assigned to methylene protons. A multiplet at $\delta 7.1-7.6 \mathrm{ppm}$ was attributed to aromatic protons. A singlet was observed for the $-\mathrm{NH}$ proton at $\delta 7.95 \mathrm{ppm}$. Compound 2 showed a singlet at $\delta 2.08 \mathrm{ppm}$ for $\mathrm{CH}_{3}$ protons. Molecular ion peaks were observed at $m / z$ 395.54, 537.92, 478.76 and 561.10 for compounds $2,7,13,19$, respectively.

Imidazoles (3-(4-(2-((1H-imidazol-2-yl)ethylamino)phenyl-2-methylquinazolin-4(3H)-one (3), 4,4'-sulfonylbis( $\mathrm{N}$-(1H-imidazol-2-yl)ethylaniline (8), $\mathrm{N}$-bis(2-1H-imidazol-2-yl)ethyl)-6-phenyl-1,3,5-triazine-2,4-diamine (14), and $\mathrm{N}$-tris(2- $1 \mathrm{H}$-imidazol-2-yl)ethyl)-1,3,5-triazine-2,4,6-triamine (20) were prepared by treating compounds 1, 6, 12 and 18 with ethylenediamine in the presence of conc. $\mathrm{HCl}$ at $160{ }^{\circ} \mathrm{C}$. The molecular ion peaks observed at $m / z$ 346.78, 435.72, 374.56 and 408.10 for compounds 3, 8, 14 and 20 confirm the molecular mass of the compounds. The IR spectrum of imidazoles (3, 8, 14 and 20) showed characteristic bands at 3050-3223 (CH), 2921-2926 $\left(\mathrm{CH}_{2}\right), 1507-1639(\mathrm{C}=\mathrm{N})$ and 3315-3402 $\mathrm{cm}^{-1}(-\mathrm{NH})$. The ${ }^{1} \mathrm{H}$ NMR spectra of all imidazole compounds showed singlets at $\delta 2.5$ and $3.4 \mathrm{ppm}$ which can be assigned to methylene protons. Compound 3 showed a multiplet at $\delta 6.7-7.5 \mathrm{ppm}$, which may be assigned to aromatic protons. The three singlets appearing at $\delta 8.2,3.37$ and $2.48 \mathrm{ppm}$ are attributed to $\mathrm{NH}$ (imidazole), methylene and methyl protons, respectively.

Benzimidazoles (3-(4-(2-(1H-benzo[ $d]$ imidazol-2-yl)ethylamino)phenyl)-2-methylquinazolin-4(3H)-one (4), 4,4'-sulfonylbis( $N$-(2-(1H-benzo[d]imidazol-2-yl)ethyl)aniline (9), $N$-bis(2-(1H-benzo[d]imidazol-2-yl)ethyl)-6-phenyl-1,3,5-triazine-2,4-diamine (15), $N$-tris(2$1 H$-benzo[d]imidazol-2-yl)ethyl)-1,3,5-triazine-2,4,6-triamine (21) were prepared by treating compounds 1, 6, 12 and 18 with o-phenylenediamine in the presence of conc. $\mathrm{HCl}$ at $160{ }^{\circ} \mathrm{C}$. Molecular ion peaks were obtained at $\mathrm{m} / \mathrm{z} 394.54,535.77,475.05$ and 558.10 for compounds 4, 9, 15 and 21, respectively. The IR spectra of benzimidazoles showed characteristic bands at 3055-3223 $(\mathrm{CH}), 2924-2930\left(\mathrm{CH}_{2}\right), 1592-1634(\mathrm{C}=\mathrm{N})$ and $3315-3411 \mathrm{~cm}^{-1}$ (-NH). The ${ }^{1} \mathrm{H}$ NMR spectrum of all the four benzimidazole compounds showed singlets at $\delta 3.3$ and $\delta 2.9 \mathrm{ppm}$, which can be assigned to methylene protons. The strong peaks observed as a singlet at $\delta 12.33$ to $12.53 \mathrm{ppm}$ are due to $\mathrm{NH}$ proton of the benzimidazole ring of compounds 15 and 21, respectively. Compound 4 showed a multiplet at $\delta 6-6.4$ ppm, which can be assigned to aromatic protons. The methyl protons attached to the quinazoline ring showed a singlet at $\delta 2.48 \mathrm{ppm}$. The singlet appearing at $\delta 8.1 \mathrm{ppm}$ can be attributed to $\mathrm{NH}$ (benzimidazole) protons.

Compounds 4,4'-sulfonylbis( $N$-(2-(1H-tetrazole-5-yl)ethyl)aniline (10), $N$-bis(2-1Htetrazol-5-yl)ethyl)-6-phenyl-1,3,5-triazine-2,4-diamine (16), and $N$-tris(2-(1H-tetrazol-5yl)ethyl)-1,3,5-triazine-2,4,6-triamine (22) were prepared by treating 3,3'-(4,4'-sulfonyl- 
bis(4,1-phenylene)bis(azanediyl))dipropanenitrile (6), 3,3'-(6-phenyl-1,3,5-triazine-2,4-diyl) bis(azanediyl)dipropanenitrile (12) and 3,3,3',1,3,5-triazine-2,4,6-triyl)tris(azanediyl)tripropanenitrile (18) with $\mathrm{NaN}_{3}$ and $\mathrm{NH}_{4} \mathrm{Cl}$ (Schemes 2-4). The infrared spectral data of all the three tetrazoles $(\mathbf{1 0}, \mathbf{1 6}$ and $\mathbf{2 2})$ revealed bands at $\mu$ 3397-3426 (-NH), 2932-3005 $\left(\mathrm{CH}_{2}\right), 1590-1655(\mathrm{C}=\mathrm{N}), 3098-3205(\mathrm{CH}), 1404-1430(\mathrm{~N}=\mathrm{N}), 1225-1231(\mathrm{~N}-\mathrm{N}=\mathrm{N})$ and 1099-1174 (tetrazole ring) $\mathrm{cm}^{-1}$. The ${ }^{1} \mathrm{H}$ NMR spectrum of the compounds showed a singlet at $\delta 2.5-2.8$ and 3.38-3.5 ppm, which can be assigned to methylene protons. The singlet observed at $\delta 5.5-5.7 \mathrm{ppm}$ was due to the $-\mathrm{NH}$ protons of the tetrazole ring. Molecular ion peaks obtained at $m / z$ 440.63, 378.78 and 414.13 for compounds 10, 16 and 22 confirm the molecular weight of the compounds.

\section{Cytotoxicity screening}

Induction of cell death or inhibition of cell proliferation is an important property for chemotherapeutic agents. The cytotoxic effect of compounds $\mathbf{2 - 4 , 7 - 1 0 , 1 3 - 1 6}$ and 19-22 was evaluated by measuring the level of cell proliferation after incubation of the cells with the test samples using the MTT colorimetric assay (10). The results are expressed as percentage of cell proliferation compared to the cell control (cells treated with vehicle,

Table III. Cytotoxic activity of newly synthesized compounds

\begin{tabular}{ccc}
\hline \multirow{2}{*}{ Compd. } & \multicolumn{2}{c}{$I C_{50}\left(\mu \mathrm{g} \mathrm{mL}^{-1}\right)$} \\
\cline { 2 - 3 } & MCF-7 & HT-29 \\
\hline $\mathbf{2}$ & 22.5 & 21.7 \\
$\mathbf{3}$ & 11.0 & 18.2 \\
$\mathbf{4}$ & 16.9 & 19.5 \\
$\mathbf{7}$ & 17.9 & 19.3 \\
$\mathbf{8}$ & 15.6 & 18.7 \\
$\mathbf{9}$ & 5.7 & 5.2 \\
$\mathbf{1 0}$ & 37.0 & 52.7 \\
$\mathbf{1 3}$ & 6.12 & 5 \\
$\mathbf{1 4}$ & 14.8 & 40.3 \\
$\mathbf{1 5}$ & 7.2 & 5.9 \\
$\mathbf{1 6}$ & 102.2 & 113.8 \\
$\mathbf{1 9}$ & 12.5 & 15.6 \\
$\mathbf{2 0}$ & 19.2 & 22.5 \\
$\mathbf{2 1}$ & 6.0 & 5.5 \\
$\mathbf{2 2}$ & 40.1 & 60.2 \\
$\mathbf{5} \mathrm{FU}$ & 6.2 & 5.5 \\
\hline
\end{tabular}

$\mathrm{IC}_{50}$ - concentration required to inhibit cell viability by $50 \%$ : Control: DMSO. 
DMSO $0.1 \%$ ). The results of $I C_{50}$ values of MCF-7 (breast cancer) and HT-29 (colon cancer) of test compounds are presented in Table III.

It is evident from the study that all the heterocyclic compounds synthesized in the present work had a cytotoxic effect on the tested cell lines. It is worth noting that compounds 9, 13, 15 and 21 exhibit $I C_{50}$ values comparable to the standard drug 5-flurouracil. From the results of the study, it is inferred that among the new heterocyclic compounds synthesized in the present research work, compounds with benzimidazole and benzoxazole moieties exhibit good cytotoxic activity. It is interesting to note that among the four benzimidazole compounds synthesized $(4,9,15$ and 21), three compounds, viz., 4,4'-sulfonylbis( $N$-(2-(1H-benzo[d]imidazol-2-yl)ethyl)aniline (9), $N$-bis(2-(1H-benzo[d]imidazol-2-yl)ethyl)-6-phenyl-1,3,5-triazine-2,4-diamine (15) and $N$-tris(2- $1 H$-benzo[ $d]$ imidazol-2-yl)ethyl)-1,3,5-triazine-2,4,6-triamine (21) exhibit potent cytotoxicity with $I C_{50}$ values of 5.7 (MCF-7), 5.2 (HT-29), 7.22 (MCF-7), 5.9 (HT-29) $6.0 \mu \mathrm{g} \mathrm{mL}^{-1}$ (MCF-7), and $5.5 \mu \mathrm{g} \mathrm{mL}^{-1}$ (HT-29), respectively. Among the four benzoxazole derivatives $(2,7,13$ and 19), N-bis(2-(benzo[d]oxazol-2-yl)ethyl)-6-phenyl-1,3,5-triazine-2,4-diamine (13), shows potent cytotoxic activity against the two cancer cell lines taken for the study. The least potency was shown by compounds 10, 16 and 22 containing tetrazole moiety.

From the data of cytotoxic activity, compounds 9, 15 and 21 were found to possess promising cytotoxic activity. This activity of the compounds may be due to the presence of dapsone, triazine and benzimidazole moieties. The remarkable antiproliferative activity of compound $\mathbf{1 3}$ may due to the presence of triazine and benzoxazole moieties.

\section{CONCLUSIONS}

In the present study, syntheses of some new benzimidazoles, benzoxazoles, imidazoles and tetrazoles is described. All the compounds were evaluated for cytotoxic activity. Benzimidazoles 9, 13, 15 and $\mathbf{2 1}$ and benzoxazole $\mathbf{1 3}$ were found to be the most active compounds with a promising cytotoxic activity. It can be deduced from the results that benzimidazole and benzoxazole moiety can create potent cytotoxic compounds, whereas imidazole and tetrazole compounds show lower cytotoxic effects. Further studies and structural modifications are needed to increase the cytotoxic activity.

Acknowledgements. - The authors thank the Management of SRM University for providing the necessary facilities to carry out the research work.

\section{REFERENCES}

1. V. P. Devmurari, P. Shivanand, M. B. Goyani, R. R. Nandanwar, N. P. Jivani and P. Perumal, Synthesis and anticancer activity of some novel 2-substituted benzothiazole derivatives, Int. J. Chem. Tech. Res. 2 (2010) 681-689.

2. R. C. Schnur, F. J. Fliri, S. Kajiji and V. A. Pollack, N-(5-fluorobenzothiazol-2-yl)-2-guanidinothiazole-4-carboxamide. A novel, systemically active antitumor agent effective against 3LL Lewis lung carcinoma, J. Med. Chem. 34 (1991) 914-918; DOI: 10.1021/jm00107a007. 
3. A. T. Balaban, D. C. Oniciu and A. R. Katritzky, Aromaticity as a cornerstone of heterocyclic chemistry, Chem. Rev. 104 (2004) 2777-2812; DOI: 10.1021/cr0306790.

4. K. Shalini, P. K. Sharma and N. Kumar, Imidazole and its biological activities: A review, Chem. Sin. 1 (2010) 36-47.

5. J. A. Kumar, A. K. Tiwari, A. Z. Ali, K. Madhusudhana, B. S. Reddy, S. Ramakrishna and B. C. Raju, New antihyperglycemic, $\alpha$-glucoside inhibitory and cytotoxic derivatives of benzimidazole, J. Enzyme Inhib. Med. Chem. 25 (2010) 80-86; DOI: 10.3109/14756360903017122.

6. B. Dahlof, R. B. Devereux and S. E. Kjeldsen, Cardiovascular morbidity and mortality in the losartan intervention for endpoint reduction in hypertension study (LIFE): a randomized trial against antenolol, Lancet 359 (2002) 995-1003; DOI: 10.1016/S0140-6736(02)08089-3.

7. T. J. Mosmann, Rapid colorimetric assay for cellular growth and survival: application to proliferation and cytotoxicity assays, J. Immunol. Meth. 65 (1983) 55-63; DOI: 10.1016/0022-1759(83)90303-4.

8. S. Ampati, S. Naik, A. R. Ganta, V. Jenugu, R. Jukanti and S. Manda, Synthesis and antiinflammatory activity of a series of novel benzoxazole derivatives, J. Pharm. Res. 3 (2010) 2444-2446.

9. S. Arulmurugan and H. P. Kavitha, 2-Methyl-3-\{4-[2-(1H-tetrazol-5-yl)ethylamino]phenyl $\}-3 H-$ quinazolin-4-one, Molbank M695 (2010) 1-5; DOI: 10.3390/M695.

10. J. Weyermann, A practical note on the use of cytotoxicity assays, Int. J. Pharm. 288 (2005) 369-376; DOI: 10.1016/j.ijpharm.2004.09.018. 